\title{
Influencia de la educación nutricional sobre la adopción de preferencias alimentarias en estudiantes de Gastronomía
}

\section{Influence of nutritional education on of food preferences for Gastronomy students}

DOI: http://dx.doi.org/10.17981/cultedusoc.11.1.2020.17

Recibido: 28/11/2019. Aceptado: 19/02/2020. Publicado: 24/03/2020

Sugey Elena Anaya García

Universidad Católica Luis Amigó. Medellín (Colombia)

sugey.anayaga@amigo.edu.co

Para citar este artículo:

Anaya, S. (2020). Influencia de la educación nutricional sobre la adopción de preferencias alimentarias en estudiantes de Gastronomía. Cultura, Educación y Sociedad, 11(1). 235-240. DOI: http://dx.doi.org/10.17981/cultedusoc.11.1.2020.17

\section{Resumen}

Los universitarios requieren un acompañamiento en educación nutricional, que les permita mantener buenos hábitos alimenticios. Esta investigación, evalúa la influencia que ejerce la educación nutricional sobre las preferencias alimentarias de los estudiantes de gastronomía, comparados con los estudiantes que no cuentan con este tipo de formación. Para ello, se emplea una muestra de 72 estudiantes de la Universidad Católica Luis Amigó (Medellín, Colombia) a los cuales se les aplica un instrumento con datos acerca de: la frecuencia de consumo de alimentos, número de comidas ingeridas a diario, horarios de alimentación, lugares de consumo alimentario diferentes al hogar, entre otros. Se encuentra que los estudiantes incluyen más en su alimentación carnes, huevos y leguminosas secas. De manera contraria, frutas y verduras no ocupan un lugar importante en sus prácticas alimentarias. Además, no se encuentran diferencias significativas entre los dos grupos de estudiantes comparados. Se concluye que el nivel de conocimiento en educación nutricional de un individuo, no es sinónimo de prácticas alimentarias saludables.

Palabras clave: Educación nutricional; costumbres alimenticias; estudiante universitario; gastronomía

\begin{abstract}
The university students require an accompaniment in nutritional education, which allows them to maintain good eating habits. This research evaluates the influence of nutritional education on food preferences of gastronomy students, compared to students who do not have this type of training. For this, a sample of 72 students from the Universidad Católica Luis Amigó (Medellín, Colombia) is used, to which an instrument is applied with data about: the frequency of food consumption, number of meals eaten daily, feeding times, places of food consumption different from the home, among others. It is found that students include more meat, eggs and dried legumes in their diet. Conversely, fruits and vegetables do not occupy an important place in their eating practices. Furthermore, no significant differences were found between the two groups of students compared. It is concluded that an individual's level of knowledge in nutritional education is not synonymous with healthy eating practices.
\end{abstract}

Keywords: Nutritional education; eating habits; university student; gastronomy 


\section{INTRODUCCIÓN}

Los hábitos alimentarios de niños y jóvenes, repercuten de manera notoria en su estado de salud en la etapa adulta, situación por la cual es necesario un acompañamiento en Educación Alimentaria y Nutricional (EAN), este tipo de educación, es necesaria cuando se pretende contribuir al mejoramiento de los hábitos alimentarios de las personas, a través de herramientas educativas que permitan su sostenimiento (Contento, 2007).

La EAN aplicada de manera correcta puede combatir la aparición de afecciones de salud, ya que "existe una alta prevalencia de factores de riesgo de enfermedades crónicas no transmisibles en los universitarios; bajo consumo de frutas y verduras, y alto consumo de grasas saturadas" (Morales, Del Valle, Soto e Ivanovic, 2013. p. 394).

Según la Encuesta Nacional de la Situación Nutricional en Colombia -ENSIN -, realizada por Profamilia, Instituto Nacional de Salud, ICBF y Ministerio de la Protección Social de Colombia (2010), uno de cada tres colombianos entre 5 y 64 años no consume frutas diariamente y cinco de cada siete del mismo rango de edad, no consume verduras en esta frecuencia.

La alimentación de los jóvenes universitarios, parece estar siendo influenciada por la disponibilidad de tiempo, por las jornadas de clases, por el factor económico, entre otros; afectando notoriamente las practicas alimentarias saludables.

El ingreso a la universidad genera una serie de cambios en la vida de los jóvenes que van desde separarse de su familia, en el caso de los estudiantes que tienen que desplazarse a las ciudades en busca de la universidad y dejar sus hogares; además de adaptarse a nuevas normas, compañeros de estudio, niveles elevados de estrés y manejo del tiempo (Barrera et al. 2008, p. 28).

El estado, la sociedad, los medios de comunicación, familia, compañeros de clase, docentes, el círculo social y otros; juegan un papel vital en el acompañamiento que los jóvenes universitarios requieren en materia de educación nutricional.

En el plan de seguridad alimentaria y nutricional del Municipio de Medellín 2016-2028, se establece como propuesta de acción, la creación de un comité, que brinde lineamientos y acompañamiento en procesos educativos y pedagógicos en alimentación y nutrición. De igual forma, el diseño e implementación de la estrategia "Educar para comer bien", con el fin de llevar hábitos alimentarios saludables a la población de esta ciudad; todo esto, para operacionalizar la política pública de seguridad alimentaria y nutricional, establecida en el 2013 en Colombia (Alcaldía de Medellín, 2015).

Ahora bien, las instituciones de educación superior en sus programas académicos de Gastronomía, deben operacionalizar el currículo a través de preparaciones alimenticias, que permitan la adopción de prácticas saludables. Sin embargo, y a pesar de que se ofrezcan, los jóvenes incurren en el inmediatismo alimenticio: productos a base de grasas saturadas, ricos en carbohidratos complejos, bajos en fibra, pobres en vitaminas y minerales; en fin, preparaciones que se alejan de lo sano y apto para el ser humano. Para Durán et al. (2012) muchas veces "el usuario no tiene opción a elegir, además, existe una amplia disponibilidad de alimentos de alto contenido en grasa y elevado valor calórico, a un precio accesible, y servidos en raciones cada vez mayores" (p. 745).

Consecuentemente, el objetivo del artículo es evaluar la influencia que ejerce la educación nutricional sobre la adopción de las preferencias alimentarias de los estudiantes de gastronomía de la Universidad Católica Luis Amigó. 


\section{Metodología}

Durante el primer semestre del 2019, se realiza la recolección de la información. Ya para la segunda mitad del año, se procede a la digitalización de la misma. El universo es la totalidad de estudiantes del pregrado de Gastronomía de la Universidad Católica Luis Amigó (Sede Medellín, Colombia). La muestra queda conformada por 72 estudiantes, distribuidos en dos grupos de 36 cada uno: el primero, que se denominó EG (Estudiantes de Gastronomía), y el otro, llamado DG (Diferentes a Gastronomía). En el cálculo de la muestra se emplea margen de error del $15 \%$.

Cómo criterios de inclusión, se tiene en cuenta, en el caso del primer grupo, estudiantes activos en la carrera a partir del año 2015 y que hayan aprobado los cursos de nutrición y dietética e identificación de alimentos. Estos, por ser asignaturas con componentes en educación nutricional. Cómo criterios de exclusión, no se consideraron aquellos estudiantes inactivos o que reprobaron los cursos antes mencionados.

El grupo DG lo conformaron estudiantes de los programas de psicología, ingeniería de sistemas y desarrollo familiar. A los estudiantes se les explica la naturaleza del estudio $y$, posterior a ello, firman el consentimiento informado. Los sujetos deciden ser parte de la investigación de manera voluntaria. El protocolo para llevar a cabo este estudio, es aprobado por el comité de ética de la Universidad Católica Luis Amigó.

Posteriormente, se aplicó un instrumento que registra datos demográficos (edad y sexo) y estrato socioeconómico. Para el análisis de las preferencias alimenticias, se tienen en cuenta los seis grupos de alimentos del plato saludable de la familia colombiana, establecido en las guías alimentarias para la población mayor de dos años, del Instituto Colombiano de Bienestar Familiar (ICBF, 2015): 1) Cereales, raíces, tubérculos y plátanos, 2) Frutas y verduras, 3) Leche y productos lácteos, 4) Carnes, huevos y leguminosas secas, y 5) Grasas y 6 . Azúcares.

El instrumento incluye indicadores, sobre: frecuencia de consumo semanal y diario por cada uno de los grupos de alimentos, inclusión semanal en la dieta de alimentos de cada grupo alimenticio, número de comidas consumidas a diario, horarios de alimentación, lugares de consumo alimentario diferentes al hogar, entre otros. Para el análisis de los datos obtenidos, se utiliza el software Excel 2016.

\section{RESUltados}

\section{Generalidades y caracterización socioeconómica}

En la Tabla 1 se observan los resultados de la caracterización socioeconómica. En cuanto al sexo de los participantes, se evidencia que en el grupo EG predominaron los hombres, con un 52,7\%, y en los DG, las mujeres, con un 55,5\%. Respecto a los estratos socioeconómicos, la mayoría de los participantes se ubica entre los niveles tres y cuatro: los EG, con un $80,5 \%$, mientras que en el grupo comparativo un $69,7 \%$. Resalta el $27,7 \%$ de este último grupo, ubicado en estratos uno y dos. Por otro lado, más del $90 \%$ de los estudiantes del grupo EG tiene menos de 25 años, mientras que, en DG, se encuntra casi un 50\% mayor a 26 años. 
TABLA 1.

Resultados de la caracterización socioeconómica

\begin{tabular}{|c|c|c|}
\hline Variable & Grupo EG (Estudiantes Gastronomía) & Grupo DG (Diferentes a Gastronomía) \\
\hline \multicolumn{3}{|c|}{ Género } \\
\hline Femenino & 47,2 & 55,5 \\
\hline Masculino & 52,7 & 44,5 \\
\hline \multicolumn{3}{|c|}{ Estrato Socioeconómico } \\
\hline $1-2$ & 5,5 & 27,7 \\
\hline $3-4$ & 80,5 & 69,4 \\
\hline $5-6$ & 13,8 & 2,7 \\
\hline \multicolumn{3}{|c|}{ Edad } \\
\hline $15-20$ & 58,3 & 13,8 \\
\hline $21-25$ & 38,8 & 36,1 \\
\hline $26-30$ & 0 & 33,3 \\
\hline$>30$ & 2,7 & 16,6 \\
\hline
\end{tabular}

*Valores dados en porcentaje (\%).

Fuente: Elaboración propia.

\section{Preferencias ALimentarias y Frecuencia de CONSUmo}

Los resultados obtenidos, en cuanto a los grupos de alimentos que más incluyen los estudiantes semanalmente en su alimentación, son: el grupo EG ubica en primer lugar carnes, huevos y leguminosas secas $(61,1 \%)$, seguidos por la leche y los productos lácteos $(44,4 \%$ y las grasas (44,4\%). Se halla, además, un porcentaje importante $(36,1 \%)$ que incluye frutas y verduras en el último lugar. El grupo DG ubica también el grupo de las carnes, huevos y leguminosas secas $(63,8 \%)$ de primero, seguido de las grasas con (41,6\%). Finalmente, los cereales, raíces, tubérculos y plátanos, y las frutas y verduras, ocupan la última posición con un $22,2 \%$.

Resulta que tanto los EG como los DG incluyen con mayor frecuencia arepa, arroz, lechuga, zanahoria, tomate, leche, huevo, aceite, mantequilla, azúcar y panela. Cabe aclarar que se integran seis alimentos por grupo alimenticio, para un total de 36. La yuca, papaya, kumis, pescado y frutos del mar, queso crema y bebida gaseosa fueron los alimentos que menos se incorporan. Las frutas ocupan un lugar intermedio.

Al preguntar acerca del consumo diario de los grupos de alimentos, el 66,6\%, tanto de los EG como los DG, incluyen el grupo de cereales, raíces, tubérculos y plátanos, de una a dos veces al día. En esa misma frecuencia, los DG incluyen grasas (50\%), frutas y verduras (47,2\%), leche y productos lácteos $(44,4 \%)$, azúcares $(38,8 \%)$ y carnes, huevos y leguminosas secas (36,1\%). Los EG incluyen frutas y verduras (55,5\%), leche y productos lácteos (50\%), grasas y azúcares (50\%) de una a dos veces al día, y de tres a cuatro veces carnes, huevos y leguminosas secas $(44,4 \%)$.

Al consultar sobre el consumo de vasos de agua al día, sólo el 33,3\% de los EG consume más de siete, el 47,2\% entre tres y seis, y el 19,4\% entre uno y dos. El 19,4\% de los DG ingiere más de siete vasos; esto quiere decir, que más del $70 \%$ consume entre uno y seis. 
Al relacionar con el número de comidas ingeridas en un día, la mayoría $(80,5 \%)$ de los EG y los DG $(88,8 \%)$ responde "entre tres y cinco". Menos del $10 \%$ consume más de cinco comidas diarias.

Respecto a los horarios de alimentación, gran parte de los EG consumen el desayuno entre las 7:00 y 9:00 am; mientras que en los DG este aspecto oscila entre 5:00 y 7:00 am. La media mañana entre las 9:00 y 11:00 ampara ambos grupos. El almuerzo, por su parte, se consume por los EG entre las 11:00 am y la 1:00 pm, y por los DG entre 1:00 y 3:00 pm. El $36,1 \%$ de los estudiantes de gastronomía no consume algo, mientras que el 50\% de los DG lo toman entre las 3:00 y 5:00 pm. La cena es consumida por ambos grupos entre las 7:00 y 9:00 pm; por último, más del 60\% de los participantes, en general, no consume merienda por la noche.

A la pregunta ¿de las comidas que consume en un día, cuántas son preparadas en su casa?, más del 50\% respondió, en ambos grupos, "entre tres y cuatro". Seguidamente, se indaga por el sitio en donde suelen consumir los alimentos que no son preparados en el hogar. El 77\% de los EG indica a la universidad como zona de consumo; mientras que el grupo comparativo manifiesta que en la universidad y en restaurantes externos $(52,7 \%)$.

\section{DisCUSIÓN Y CONCLUSIONES}

En el artículo se evidencian resultados relevantes, relacionados con la influencia, o no, de la educación nutricional para la adopción de preferencias alimentarias en los estudiantes. En cuanto a los grupos de alimentos que más incluye la población objetivo en su alimentación semanal, se ubican las carnes, huevos y leguminosas secas de primero y, de último, el grupo de frutas y verduras.

En el caso de las frutas y verduras, no parece importante su inclusión en la alimentación de los estudiantes. Con relación a los alimentos de mayor aceptación, teniendo en cuenta los grupos alimenticios establecidos en Colombia, no hay grandes diferencias. La yuca, papaya, pescado y frutos del mar y las bebidas gaseosas fueron los alimentos que menos incluyen. El consumo de agua fue ligeramente mayor en el grupo EG; sin embargo, un gran número de estudiantes no llega a la recomendación diaria entre "cuatro y seis vasos de agua adicionales a los líquidos contenidos en el resto de la alimentación” (ICBF, 2015. p. 23).

Respecto al número de comidas incluidas en un día, ambos grupos señalan en similar porcentaje: Tres a cinco. Para los horarios de alimentación, el desayuno y almuerzo varían entre un grupo y otro. Esto se puede explicar por la diferencia generacional, en donde personas mayores a 26 años, en el grupo comparativo, evidencian una mayor vinculación laboral. La universidad y los restaurantes constituyen los lugares de alimentación, diferentes al hogar, en donde ambos grupos de estudiantes consumen sus alimentos. En promedio, sólo tres de las comidas son preparadas al interior de sus familias.

En conclusión, al evaluar si la educación alimentaria y nutricional influye sobre las preferencias alimentarias, no se encuentran grandes diferencias entre el grupo que recibe dicha formación y los que no. Aunado a lo anterior, se sugiere realizar otros estudios que den cuenta de los factores que influencian notoriamente las prácticas alimentarias inadecuadas en los jóvenes universitarios, ya que esta investigación evidencia que el nivel de conocimiento en educación nutricional que tenga un individuo, no es sinónimo de prácticas alimentarias saludables. 


\section{Agradecimientos}

A la Universidad Católica Luis Amigó, Medellín, Colombia, quién financió y apoyó el proyecto de investigación del cual deriva el presente artículo.

\section{REFERENCIAS}

Alcaldía de Medellín. (2015). Plan de Seguridad Alimentaria y Nutricional del Municipio de Medellín 2016-2028. [Online]. Disponible en https:/www.medellin.gov.co/irj/go/ km/docs/pccdesign/SubportaldelCiudadano_2/PlandeDesarrollo_0_15/InformacinGeneral/Shared\%20Content/Documentos/instrumentos/ps/PLAN_SEGURIDAD_ALIMENTARIA_2016-2028.pdf

Barrera, J., Bicenty, A., Gerena, R., Ibañez, É., Martínez, J. y Thomas, Y. (2008). Cambios de hábitos alimentarios de los estudiantes de odontología de la Fundación Universitaria San Martín de Bogotá, Colombia. Nova, 6(9), 27-34. Disponible en https://hemeroteca.unad.edu.co/index.php/nova/article/view/393

Contento, I. R. (2007). Nutrition education. Linking research, theory and practice. Sudbury: Jones and Bartlett Publishers.

Durán, S., Bazaez, G., Figueroa, K., Berlanga, M. R., Encina, C. y Rodríguez, M. P. (2012). Comparación en calidad de vida y estado nutricional entre alumnos de nutrición y dietética y de otras carreras universitarias de la Universidad Santo Tomás de Chile. Nutrición Hospitalaria, 27(3), 739-746. http://dx.doi.org/10.3305/nh.2012.27.3.5746

Morales, G., Del Valle, C., Soto, A. e Ivanovic, D. (2013). Factores de riesgo cardiovascular en estudiantes universitarios. Revista chilena de nutrición, 40(4), 391-396. http:// dx.doi.org/10.4067/S0717-75182013000400010_

República de Colombia. ICBF. (2015). Guías Alimentarias Basadas en Alimentos para la población colombiana mayor de 2 años. [Manual para facilitadores]. Disponible en https://www.icbf.gov.co/sites/default/files/manual_facilitadores_gaba.pdf

República de Colombia. Profamilia, Instituto Nacional de Salud, ICBF y Ministerio de la Protección Social. (2010). Encuesta Nacional de la Situación Nutricional en Colombia. ENSIN 2010. [Online]. Disponible en https://www.icbf.gov.co/sites/default/files/resumenfi.pdf 